\title{
Metastatic Spinal Cord Compression as the First Manifestation of Malignancy - A Retrospective Study of Surgical Outcome from Single Institution Spine Tumor Registry
}

Ue-Cheung Ho

National Taiwan University Hospital

Dar-Ming Lai

National Taiwan University Hospital

Fu-Ren Xiao

National Taiwan University Hospital

Shih-Hung Yang

National Taiwan University Hospital

Chang-Mu Chen

National Taiwan University Hospital

Fon-Yih Tsuang ( $\nabla$ tsuangfy@gmail.com )

National Taiwan University Hospital

\section{Research Article}

Keywords: Metastatic Spinal Cord Compression, Manifestation of Malignancy, Surgical Outcome, Spine Tumor Registry, Single Institution

Posted Date: June 9th, 2021

DOI: https://doi.org/10.21203/rs.3.rs-574132/v1

License: (c) (i) This work is licensed under a Creative Commons Attribution 4.0 International License. Read Full License 


\section{Abstract}

Given the limited studies addressing the issue about the effect of different surgical modalities for metastatic spinal cord compression (MSCC) as the first malignancy manifestation, we conducted a retrospective case-control study to evaluate the surgical outcome of MSCC as the first malignancy manifestation. A total of 59 patients who were suspected of having metastatic spinal cord compression and underwent surgery from 2008 to 2017 were enrolled in the study. All patients were categorized into either 'debulking group' or 'palliative group'. The primary outcome was progression-free survival (PFS). The secondary outcomes were overall survival (OS), Frankel scale, and Karnofsky scores. All the outcomes were analyzed with a data cutoff of December 31, 2017. There was a significant difference between groups in progression-free survival (PFS) $(p=0.0036)$. However, there was no significant difference between groups in the overall survival (OS) $(p=0.8669)$. Age of onset, gender, duration of symptoms, and location of spinal metastasis, initial Frankel, initial Tomita scores, and initial Karnofsky performance scale showed no significant differences between groups. In conclusion, debulking surgery was shown to provide better neurological recoveries and could be considered first in patients with metastatic spinal cord compression as the first malignancy manifestation.

\section{Introduction}

The spine is a common site for metastasis, and the most common causes of metastases are breast, lung, and prostate cancer. ${ }^{1}$ The incidence rate of spinal metastasis has increased recently because of advances in multidisciplinary treatment that have led to longer survival rates. ${ }^{2}$ The manifestation of spinal metastasis can range from simple low back pain to devastating spinal cord compression, which might cause permanent neurological impairment. Metastatic spinal cord compression (MSCC) is an oncological emergency that calls for immediate surgical intervention whenever indicated, especially for those with mechanically unstable spines. Epidemiological studies have reported that MSCC could be the initial presentation of malignancy in up to $20 \%$ of cases. ${ }^{3-5}$ In certain circumstances, primary cancer could not initially be identified. These patients should be considered as treatment naïve and a distinct group from those with known primary cancer. The initial assessment of a patient with spine metastasis is important for guiding treatment and should incorporate performance status, systemic burden of disease, control of systemic disease, and systemic treatment options. ${ }^{2}$ This type of assessment does not apply for those with unknown primary cancer because tumor status cannot initially be evaluated. In addition, there is no time for comprehensive survey due to the neurological emergency that necessitates immediate surgical intervention. The surgical outcome for those presenting with MSCC due to cancers of unknown primary origin has been reported in several studies ${ }^{6-9}$; however, the definition of spinal metastasis with cancer of unknown primary origin is not always consistent. Only a few studies addressing the issue about the effect of different surgical modalities for treating these populations exists. Given the limited information available until now regarding the outcome of different surgical approaches in patients with MSCC as the first malignancy manifestation, a comprehensive evaluation of the surgical role is 
necessary. The aim of this study was to evaluate the surgical outcome of MSCC as the first malignancy manifestation.

\section{Methods}

\section{Data Source and Cohort Selection}

In a National Taiwan University Hospital (NTUH) Research Ethics Committee (REC) approved protocol (\#201810005RIND), a retrospective analysis of six-hundred twenty-eight consecutive patients who were suspected of having MSCC after presenting with neck and/or back pain or neurological deficits and had previous surgeries at the National Taiwan University Hospital (NTUH) between 2008 and 2017 and its affiliated branch, Yun-Lin, between 2014 and 2017 were enrolled in the spinal oncology registry. The inclusion criteria were confirmation of spinal metastasis by computed tomography (CT) or magnetic resonance imaging (MRI), surgery for spinal metastasis with available specimen confirmed to be malignant by a pathologist, no previous history of cancer, and no history of radiotherapy and/or chemotherapy, hormone and target therapy, and/or surgery for spinal metastasis. The exclusion criteria included a lack of spinal cord compression, previously known primary cancer, and final diagnosis other than spinal metastasis. A total of 59 patients were enrolled in this study and were grouped based on the surgical approaches for spinal metastasis (Fig. 1). Patients undergoing intralesional corpectomy with reconstruction and fixation were classified as the 'debulking group'; on the other hand, those patients undergoing palliative decompressive laminectomy with/without fixation were classified as the 'palliative group'. The decision for surgery was at the discretion of treating surgeon.

Patient demographic and clinical characteristics, including the age of onset, gender, duration of symptoms, location of spinal metastasis, initial Frankel scale, initial Tomita score, and initial Karnofsky performance scale (KPS), were examined retrospectively. The parameter for primary cancer from the Tomita score was based on the pathology obtained after MSCC surgery. If the pathology showed malignancy and no primary site of cancer could be found after an extensive investigation, a cancer of unknown primary would be allocated in each scoring system. All the patients received adequate adjuvant chemotherapy, radiotherapy, hormone therapy, or target therapy per protocol.

In our institute, all admitted patients have provided a universal informed consent the data might be used for educational or academic purpose in the future. This retrospective study was approved by National Taiwan University Hospital (NTUH) Research Ethics Committee (REC) protocol (\#201810005RIND) and was adhered to the guidelines described in the Helsinki Declaration for medical research involving human subjects. After the approval for the retrospective study, the authors could obtain the access to the electronic data, which was extracted anonymously from the Spine Tumor Registry database in accordance with the Taiwan's government regulation. The patient privacy was protected and maintained throughout the study. We confirmed that all methods were performed in accordance with the relevant guidelines and regulations.

\section{Outcome}


The primary outcome was progression-free survival (PFS) assessed by the investigator. PFS was defined as the time from surgery to the earlier detection of neurological deterioration. The secondary outcome was overall survival (OS). OS was defined as the time from surgery to death due to any cause and was analyzed with a data cutoff of December 31, 2017. The patients' Frankel scale and Karnofsky performance scale were also assessed three months after surgery. If the patient had deteriorated postsurgical neurological function that led to a decrease in Frankel scale or Karnofsky performance scale score, they were specified to have had a neurological adverse event.

\section{Statistical Analysis}

Statistical analyses were performed with SPSS software. Continuous data were compared using the Student's $t$ test, and categorical data were compared by the Fisher's exact and the Pearson chi-squared tests where appropriate. Association analyses were conducted using logistic regression. Kaplan-Meier cumulative survival curves for the primary and secondary outcomes were used to compare patient cohorts. The log-rank test was used to assess differences in survival curves. An asterisk indicated significant differences $\left({ }^{*} p<0.05\right)$.

\section{Results}

From 2008 to 2017, a total of 59 patients who underwent surgery for MSCC as the first malignancy manifestation were included for investigation. The palliative group consisted of 38 patients and 21 patients formed the debulking group (Table 1). The average ages were 67.53 and 65.62 years for the palliative and debulking groups, respectively. Most cases in both groups presented with thoracic spinal metastases followed by lumbar and cervical spine metastases. The majority of patients presented with paraparesis and back pain. No significant differences were observed between the two groups regarding pre-operative Frankel and Karnofsky performance scale scores and symptom duration. The patients in the palliative group were more likely to have lower pre-operative Tomita scores than those in the debulking group. The primary cancer site could be identified in most of the cases after the surgery and was equally distributed in both groups with non-small cell lung cancer being the leading cause followed by breast and prostate cancers, hepatocellular carcinoma, and others (Table 2). There was only one case of pathology reporting poorly differentiated carcinoma, and no primary site of cancer could be identified after serial imaging studies and immunohistochemistry staining.

Karnofsky performance and Frankel scales (Figs. 2 and 3, respectively) in both groups improved three months after the operation; however, the difference between both groups did not achieve statistical significance. Three patients presented neurological deterioration, and all of them were in the palliative group.

The analysis concerning neurological adverse events at one and three months after the operation is presented in Table 3. All variables, including the age of onset, gender, duration of symptoms, location of spinal metastasis, initial Frankel, Tomita scores, and Karnofsky performance scale scores were insignificant. 
Progression-free survival (PFS) between the groups was significantly different with longer survival in the debulking group (Fig. 4). No significant differences in OS between groups were noted although there was a trend toward longer survival in the debulking group (Fig. 5).

\section{Discussion}

Epidemiological studies have reported that MSCC could be the initial presentation of malignancy up to $20 \%,{ }^{3-5}$ and it can occur in any cancer type. ${ }^{10-12}$ Regardless of whether the site of the primary cancer is known or not, surgery does play a role in spinal metastases management because MSCC is an oncological emergency that necessitates immediate surgical intervention. According to a recent review, an integrated multidisciplinary approach is needed for the management of spinal metastases due to its complexity. ${ }^{2}$ Before selecting an appropriate surgical approach, it is crucial to have an initial assessment of patient performance status, systemic burden of disease, and systemic treatment options. If the patient has very poor performance status, extensive systemic disease, or lack of available systemic treatment, these factors might preclude the possibility of better neurological recovery after the operation.

Two prognostic scoring systems, Tomita score and revised Tokuhashi score, are used to guide the management of spinal metastases. ${ }^{13-14}$ However, they are not practical in the setting of MSCC due to the lack of time for extensive surveys. The treating surgeon first encounters the issue as to whether the patient should undergo surgery. If surgery is indicated, the treating surgeon has to decide which surgical approach is appropriate.

There are a few articles that have addressed the issue. The first report of a surgical series concerning patients with an unknown source of spinal metastases found that select patients could benefit from aggressive surgical intervention. ${ }^{6}$ However, the extent of resection had no effect on survival or local recurrence. The population in this study was heterogeneous because they included patients who had a prior diagnosis of malignancy and subsequent treatment. In addition, this study included all the patients with spinal metastases, which might be different from MSCC. Two other studies published in the United Kingdom and Sweden analyzed patients presenting acutely with MSCC due to an unknown primary cancer. $^{7-8}$ It has been reported that most patients underwent posterior fixation and stabilization, and they had similar complication rates, hospitalization durations, and neurological outcomes as those patients with a known primary. Postoperative survival was dependent on the type of primary tumor, and patients with cancers of unknown primary had the worst prognosis. This finding supported the recommendation that the surgery was safe and should be done as soon as possible. However, both studies do not compare the surgical outcome of different surgical approaches. The latest article published in China compared the surgical outcomes of different modalities in patients with spinal metastasis from cancer of unknown primary. ${ }^{9}$ The article described a better neurological recovery and quality of life for the patient after surgery. Within that group, the patients could benefit from more aggressive approaches. Of note, this study does investigate the surgical outcomes of patients with cancer of unknown primary whose primary site of cancer could not be found even after a detailed survey. Overall, there is limited literature to address 
the issue about the outcomes of different surgical approaches in patients with MSCC as first the manifestation of malignancy.

Our anatomical distribution of spinal metastasis was in agreement with previous studies with most cases being in the thoracic spine followed by lumbar and cervical spine. The initial Karnofsky performance and Frankel scales in both groups were better than those in most of the previous reports. This finding might be the result of rapid medical accessibility and early referral from the treating physician due to awareness of the spinal emergency. Both Karnofsky performance and Frankel scales in each group improved three months after the operation, a finding that agreed with those of previous studies in which surgery was warranted and could maintain and improve ambulatory function in patients with spinal metastasis or MSCC as the first manifestation of malignancy. We found only three patients in our cohort who had neurological deterioration after the operation. All of them were in the palliative group, one with debilitating back pain refractory to medication, another with post-operative stroke with hemiplegia, and the other with Brown-Séquard syndrome. To compare the outcomes of different surgical approaches in these patients, we analyzed survival curves from the end of the latest follow-up. There was a significant difference in PFS between the two groups with longer survival in the debulking group. However, there was no significant difference in OS between groups. The analysis concerning neurological adverse events at one and three months after the surgery revealed that the outcome was not related to variables other than surgery, which indicated that different surgical modalities accounted for different surgical outcomes. Of note, the initial Tomita score in the debulking group was higher than that in the palliative group. It is counterintuitive to the prognostic model based on the Tomita score because the present study showed that surgical outcomes were better in the debulking group. Thus, a more aggressive approach was warranted in managing patients with metastatic spinal cord compression as the first manifestation of malignancy.

Cancers of unknown primary are defined as histologically proven cancers whose primary sites cannot be identified after extensive investigation. ${ }^{15-16}$ Prognoses for these patients are not as optimistic as those with known primary cancer because of their aggressiveness, early dissemination, and unpredictable nature. ${ }^{17-18}$ Despite systemic treatment, the median OS of the patients with cancers of unknown primary is about 6 to 12 months. ${ }^{6-7,18}$ Our study demonstrated that these patients could benefit from debulking surgery if the cancer type was unknown when the surgeon made the decision to perform surgery. However, the importance of a thorough evaluation in order to identify the primary tumor cannot be overemphasized. Those patients with known primary cancer can have adequate systemic therapy that significantly improves OS over those with cancers of unknown primary. The outcome of improving both Karnofsky performance and Frankel scale scores after the operation means that these patients have the opportunity to rapidly proceed to systemic therapy and thus, have longer survival. In addition, the median time from a patient's arrival at our hospital to the operating room is about 12 hours in each group (minimum six hours, maximum 120 hours), which is much shorter than in other countries. ${ }^{7,19}$ One study published in the United Kingdom reported that earlier surgery could provide better neurological recovery ${ }^{19}$; however, the effect was not significant, and most of the patients underwent surgery 48 hours after the 
arrival. Further studies are needed to clarify whether earlier surgery could provide more neurological recoveries and longer survival. In summary, our findings highlight the importance of a more aggressive surgical approach in managing patients with metastatic spinal cord compression as the first manifestation of malignancy.

The present study has several limitations. First, this was a retrospective study. The patients were not randomized, and the surgical approaches were chosen at the discretion of the treating physicians. Second, the patients who did not have neurological deterioration tended to be lost to follow-up during the study period. This may have biased our surgical outcome. Third, our sample size was small. Each cancer type has its own disease nature and treatment response; however, we did not incorporate cancer type into the analysis due to the small sample size. All of the surgery- and instrument-related fees were covered by National Health Insurance $(\mathrm{NIH})$, but a prosthesis, such as titanium wire mesh or expandable body cage, used for vertebral body reconstruction was not. This factor might have also biased the results.

In conclusion, surgery can improve the neurological outcome of spine metastasis as the first manifestation of malignancy, and debulking surgery was shown to provide better neurological recoveries and OS. Thus, debulking surgery rather than palliative surgery can be considered first when clinical information is limited and a patient is a suitable candidate for aggressive surgery.

\section{Declarations}

\section{Acknowledgements}

We acknowledge the statistical assistance provided by the Taiwan Clinical Trial Statistical Center and the consultation by the Professor Chin-Hao Chang.

\section{Author contributions}

U.C.H., F.Y.T., wrote the main manuscript and prepared the figures and tables. U.C.H. analyzed and interpreted data. D.M.L., F.R.X., S.H.Y., C.M.C. created the concept and design of the work and reviewed the manuscript.

\section{Competing interests}

We declare that we have no competing interests in the authorship or publication of this contribution.

\section{References}

1. Ropper, A. E. \& Ropper, A. H. Acute Spinal Cord Compression. N Engl J Med 376, 1358-1369 (2017).

2. Spratt, D. E. et al. An integrated multidisciplinary algorithm for the management of spinal metastases: an International Spine Oncology Consortium report. The Lancet Oncology 18, e720-e730 (2017). 
3. Boussios, S. et al. Metastatic Spinal Cord Compression: Unraveling the Diagnostic and Therapeutic Challenges. Anticancer Res 38, 4987-4997 (2018).

4. Lawton, A. J. et al. Assessment and Management of Patients With Metastatic Spinal Cord Compression: A Multidisciplinary Review. Journal of clinical oncology : official journal of the American Society of Clinical Oncology 37, 61-71 (2019).

5. Prasad, D. \& Schiff, D. Malignant spinal-cord compression. The Lancet. Oncology 6, 15-24 (2005).

6. Aizenberg, M. R. et al. Surgical management of unknown primary tumors metastatic to the spine. $J$ Neurosurg Spine 16, 86-92 (2012).

7. Quraishi, N. A. et al. Metastatic spinal cord compression as a result of the unknown primary tumour. Eur Spine J 23, 1502-1507 (2014).

8. Wanman, J. et al. Metastatic spinal cord compression as the first sign of malignancy. Acta orthopaedica 88, 457-462 (2017).

9. Ma, Y. et al. Quality of Life of Patients with Spinal Metastasis from Cancer of Unknown Primary Origin: A Longitudinal Study of Surgical Management Combined with Postoperative Radiation Therapy. The Journal of bone and joint surgery. American volume 99, 1629-1639 (2017).

10. Liaukovich, M., Wu, S., Yoon, S., Schaffer, J. \& Wang, J. C. Hepatocellular carcinoma presenting as spinal cord compression in Native Americans with controlled hepatitis C: two case reports. Journal of medical case reports 12, 282 (2018).

11. Salazar-Mejia, C. E. et al. Malignant Spinal Cord Compression Syndrome as an Initial Presentation of Testicular Cancer. Case reports in oncological medicine 2018, 5757434 (2018).

12. Stein, A. A. et al. Myelopathy from Intradural Extramedullary Metastasis as an Initial Presentation of Metastatic Melanoma. Cureus 10, e2668 (2018).

13. Tomita, K. et al. Surgical strategy for spinal metastases. Spine 26, 298-306 (2001).

14. Morgen, S. S. et al. A revision of the Tokuhashi revised score improves the prognostic ability in patients with metastatic spinal cord compression. Journal of cancer research and clinical oncology 144, 33-38 (2018).

15. National Collaborating Centre for, $\mathrm{C}$. in Diagnosis and Management of Metastatic Malignant Disease of Unknown Primary Origin (National Collaborating Centre for Cancer (UK). National Collaborating Centre for Cancer., 2010).

16. Losa, F. et al. SEOM clinical guideline on unknown primary cancer (2017). Clinical \& translational oncology : official publication of the Federation of Spanish Oncology Societies and of the National Cancer Institute of Mexico 20, 89-96 (2018).

17. Pavlidis, N. Cancer of unknown primary: biological and clinical characteristics. Annals of oncology : official journal of the European Society for Medical Oncology 14 Suppl 3, iii11-18 (2003).

18. Pavlidis, N. \& Pentheroudakis, G. Cancer of unknown primary site. Lancet (London, England) 379, 1428-1435 (2012). 
19. Quraishi, N. A. et al. Effect of timing of surgery on neurological outcome and survival in metastatic spinal cord compression. Eur Spine J 22, 1383-1388 (2013).

\section{Tables}

Table 1

Demographic data of enrolled patients

\begin{tabular}{|llll|}
\hline & Palliative surgery & Debulking surgery & p value \\
\hline Age (year) (mean, std) & $\mathrm{N}=38$ & $\mathrm{~N}=21$ & \\
\hline Sex (n, \%) & $67.53(13.40)$ & $65.62(15.24)$ & 0.62 \\
\hline Male & & & 0.17 \\
\hline Female & $25(65.79)$ & $10(47.62)$ & \\
\hline Location (n, \%) & $13(34.21)$ & $11(52.38)$ & 0.56 \\
\hline Cervical & & & \\
\hline Thoracic & $4(10.53)$ & $4(19.05)$ & 0.54 \\
\hline Lumbar & $30(78.95)$ & $16(76.19)$ & \\
\hline Initial Frankel (n, \%) & $4(10.53)$ & $1(4.76)$ & \\
\hline A & & & 0.527 \\
\hline B & $0(0)$ & $0(0)$ & \\
\hline C & $2(5.26)$ & $0(0)$ & \\
\hline D & $11(28.95)$ & $4(19.05)$ & \\
\hline E & $13(34.21)$ & $7(33.33)$ & 10.27 \\
\hline Initial KPS (mean, std) & $12(31.58)$ & $10(47.62)$ & \\
\hline Symptom duration (month) (mean, std) & $73.16(15.44)$ & $77.62(13.75)$ & \\
\hline Initial Tomita* (mean, std) & $1.46(1.61)$ & $1.38(1.70)$ & \\
\hline Time from arrival to operation (hour) (mean, std) & $31.89(38.40)$ & $38.85(36.64)$ & \\
\hline * The parameter for primary cancer from the Tomita score was based on the pathology obtained after & \\
\hline MSCC surgery. & & & \\
\hline
\end{tabular}


Table 2

Cancer types of enrolled patients

\begin{tabular}{|lll|}
\hline Cancer & Palliative surgery & Debulking surgery \\
\hline & $\mathrm{N}=38$ & $\mathrm{~N}=21$ \\
\hline Non-small cell lung cancer & 19 & 8 \\
\hline Breast cancer & 4 & 5 \\
\hline Prostate cancer & 6 & 2 \\
Hepatocellular carcinoma & 2 & 2 \\
\hline Others & 7 & 3 \\
\hline Unknown & 0 & 1 \\
\hline
\end{tabular}

Table 3

Univariate analysis of adverse events one and three months after the operation

\begin{tabular}{|c|c|c|c|c|}
\hline \multirow[b]{2}{*}{ Variables } & \multicolumn{2}{|c|}{ One month after the operation } & \multicolumn{2}{|c|}{ Three months after the operation } \\
\hline & OR $(95 \% \mathrm{Cl})$ & $\mathrm{p}$ value & OR $(95 \% \mathrm{Cl})$ & $\mathrm{p}$ value \\
\hline Age & $1.02(0.96-1.08)$ & 0.56 & $1.01(0.97-1.06)$ & 0.58 \\
\hline Sex & $1.42(0.24-8.44)$ & 0.70 & $1.48(0.39-5.61)$ & 0.56 \\
\hline \multicolumn{5}{|l|}{ Location } \\
\hline Thoracic vs cervical & NA & 0.97 & NA & 0.96 \\
\hline Lumbar vs cervical & $0.85(0.09-8.45)$ & 0.89 & $0.83(0.15-4.78)$ & 0.84 \\
\hline \multicolumn{5}{|l|}{ Initial Frankel } \\
\hline E vs $B$ & NA & 0.98 & NA & 0.98 \\
\hline E vs C & $0.45(0.04-4.82)$ & 0.51 & $2.21(0.21-23.55)$ & 0.51 \\
\hline E vs D & $0.70(0.11-4.71)$ & 0.72 & $1.42(0.21-9.52)$ & 0.72 \\
\hline Initial KPS & $1.03(0.96-1.10)$ & 0.37 & $1.03(0.98-1.08)$ & 0.25 \\
\hline Symptom duration (month) & $0.72(0.32-1.60)$ & 0.42 & $1.19(0.83-1.69)$ & 0.35 \\
\hline Initial Tomita & $1.10(0.72-1.68)$ & 0.67 & $0.94(0.68-1.31)$ & 0.72 \\
\hline
\end{tabular}

\section{Figures}


Total patients $(\mathrm{N}=628)$

\begin{tabular}{|l|l|}
\hline & \begin{tabular}{l} 
No spinal cord compression $(\mathrm{N}=28)$ \\
\hline Known primary cancer $(\mathrm{N}=495)$
\end{tabular} \\
\hline $\begin{array}{ll}\text { Primary cancer is unknown prior } \\
\text { to surgical intervention }(\mathrm{N}=59)\end{array}$ & $\begin{array}{l}\text { Diagnosis other than metastasis }(\mathrm{N}=46) \\
\text { Glioma }(\mathrm{N}=3) \\
\text { Ependymoma }(\mathrm{N}=9) \\
\text { Meningioma }(\mathrm{N}=1) \\
\text { Hematological malignancy }(\mathrm{N}=18) \\
\text { Giant cell tumor }(\mathrm{N}=2) \\
\text { Benign lesion }(\mathrm{N}=3) \\
\text { Peripheral nerve tumor }(\mathrm{N}=4) \\
\text { Malignant thabdoid tumor }(\mathrm{N}=1) \\
\text { Neuroendocrine tumor }(\mathrm{N}=5)\end{array}$ \\
\hline
\end{tabular}

Figure 1

Flow chart of patient enrollment 


\section{Karnofsky score}

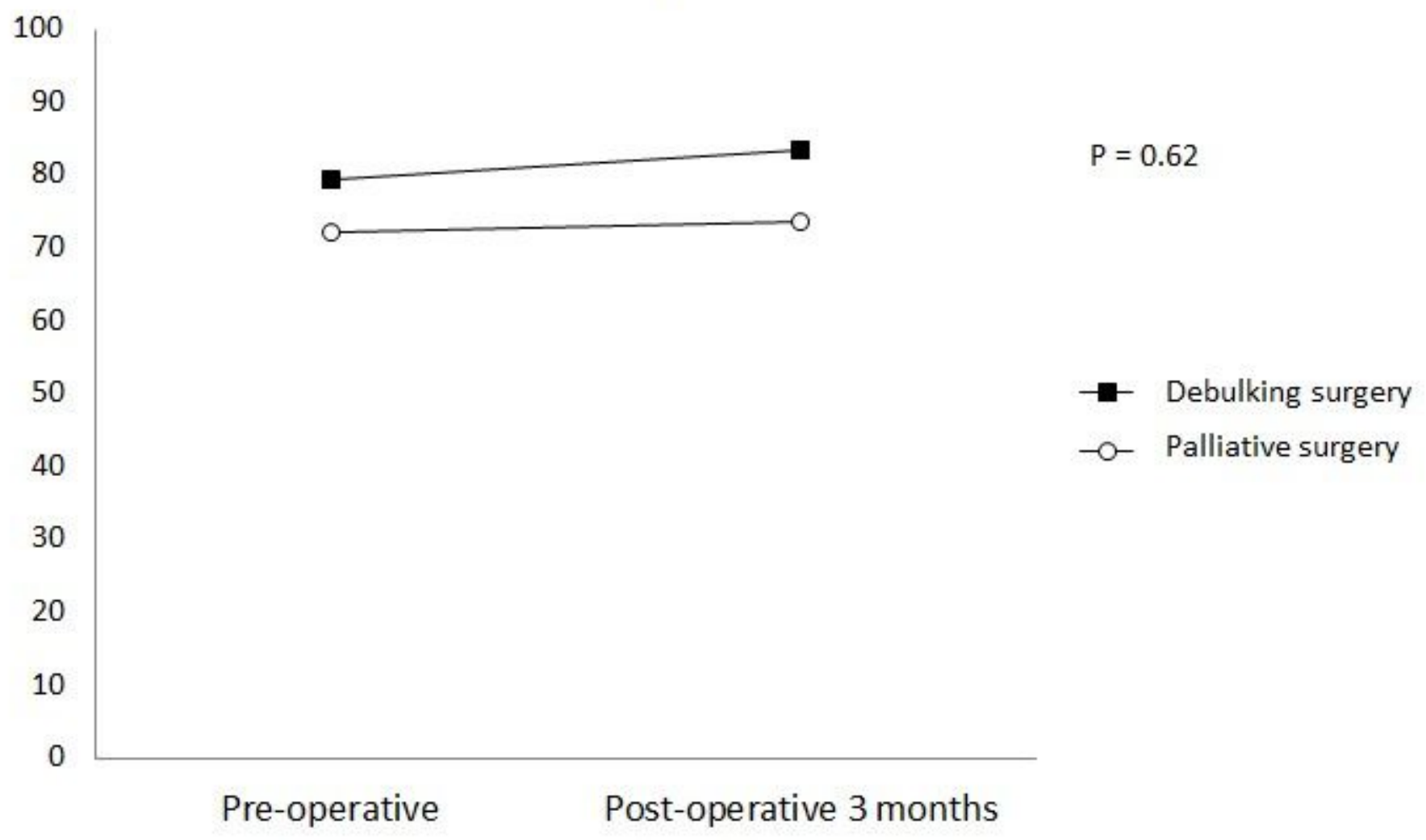

Figure 2

Preoperative and three-month post-operative Karnofsky scores for palliative and debulking groups

Debulking surgery

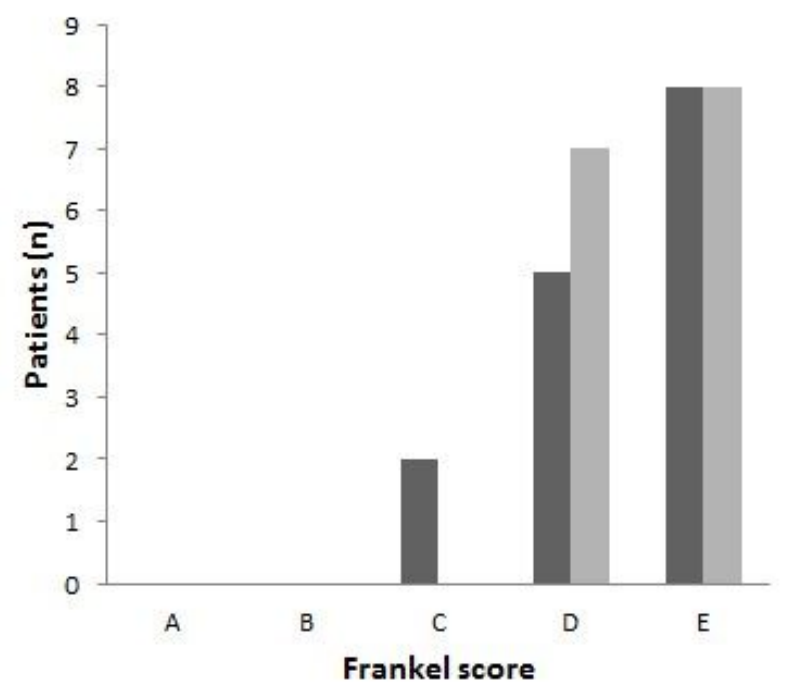

\section{Palliative surgery}

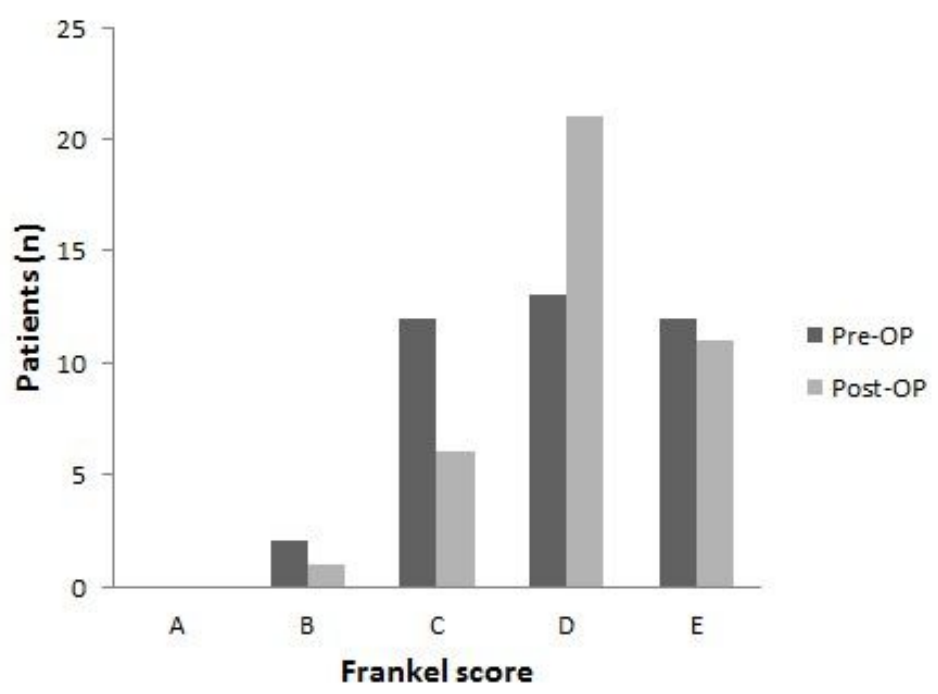

Figure 3

Comparison between preoperative and postoperative Frankel scores in palliative and debulking groups 


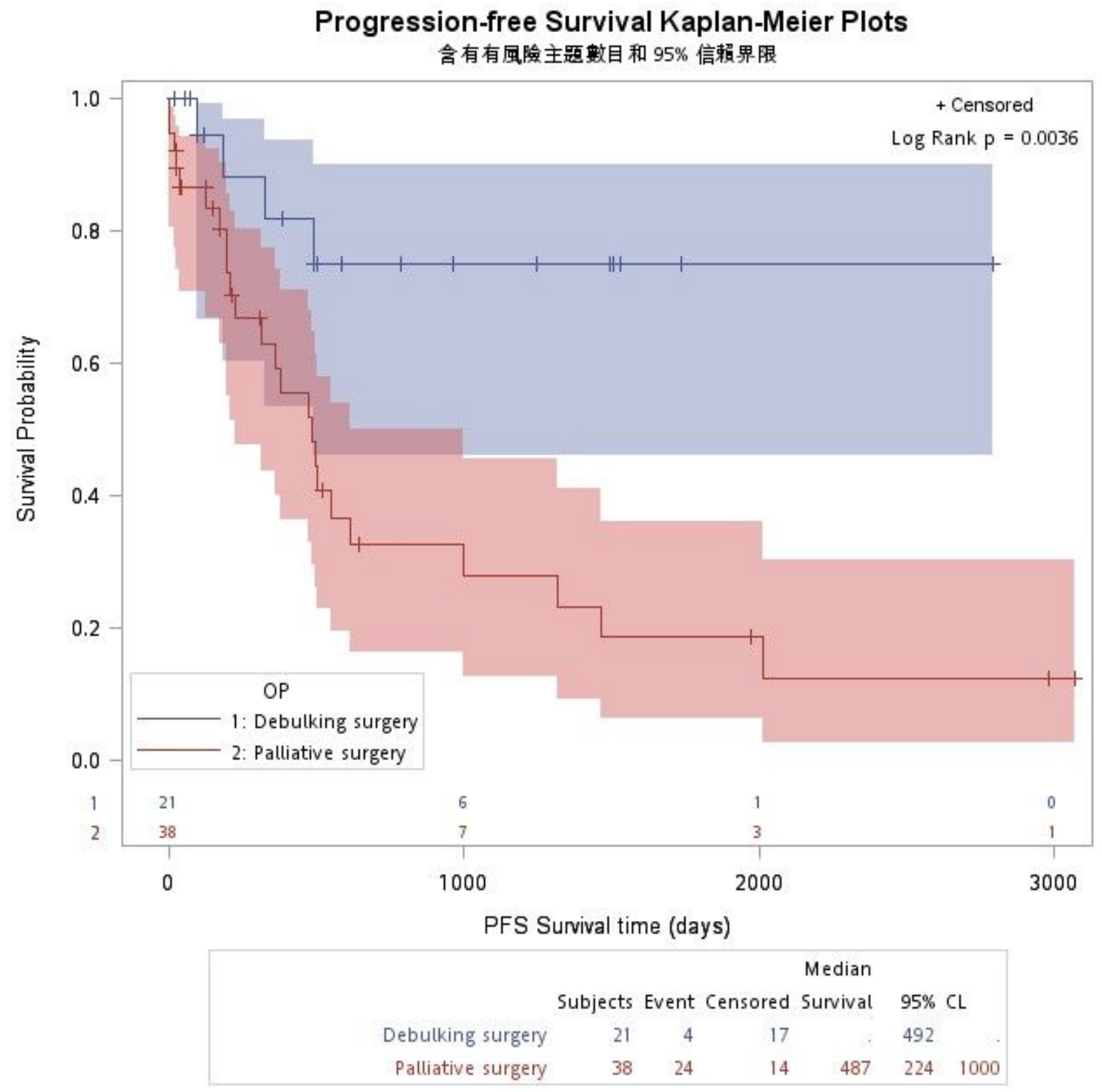

Figure 4

Kaplan-Meier curve for progress-free survival (PFS) 


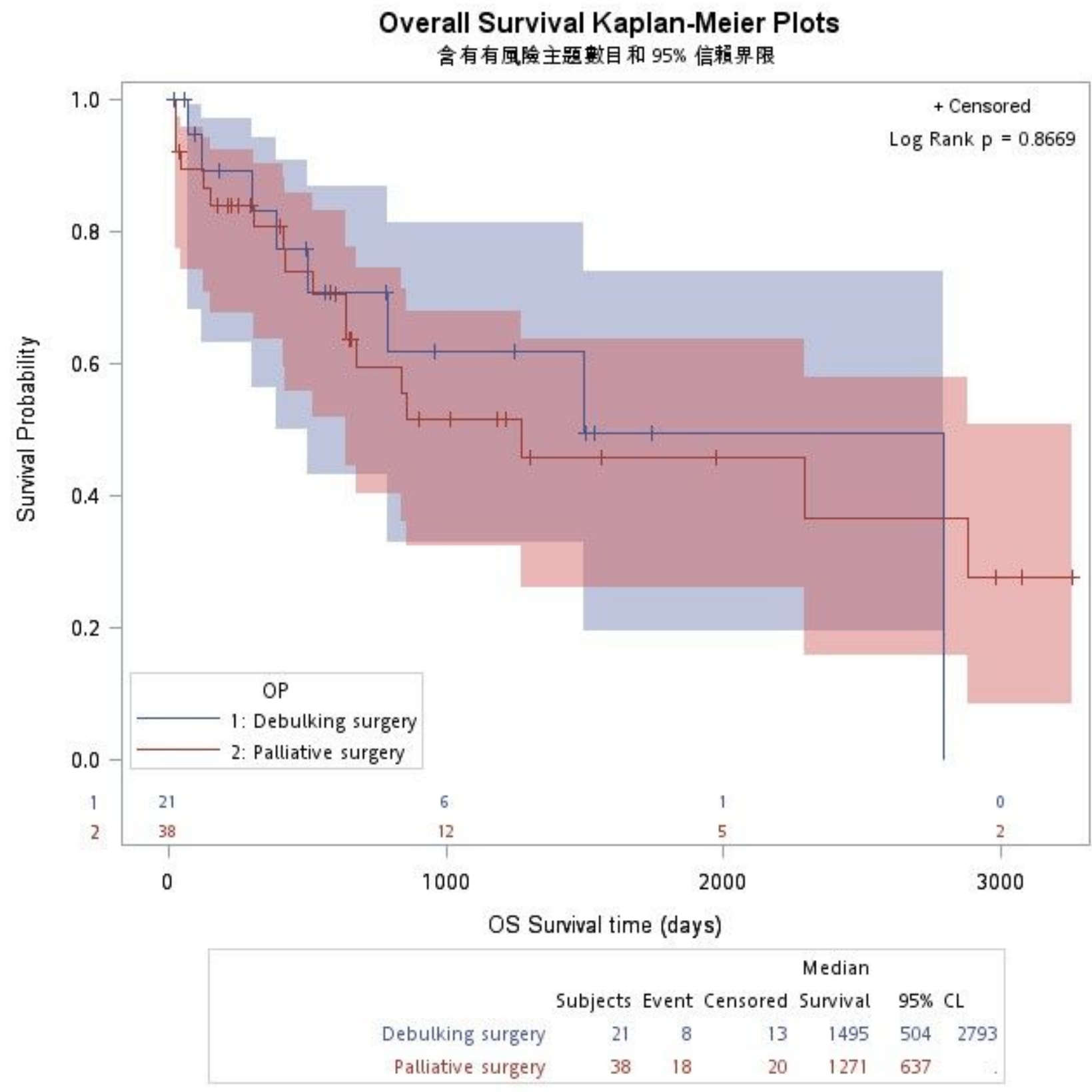

Figure 5

Kaplan-Meier curve for overall survival (OS) 